九州大学学術情報リポジトリ

Kyushu University Institutional Repository

\title{
Parametric instabilities of circularly polarized Alfve' $n$ waves in a relativistic electron-positron plasma
}

Matsukiyo, Shuichi

Department of Earth System Science and Technology, Kyushu University

Hada, Tohru

Department of Earth System Science and Technology, Kyushu University

http://hdl. handle. net/2324/16868

出版情報: Physical review. Third series. E, Statistical, nonlinear, and soft matter physics. 67 (4), pp.046406-, 2003. APS

バージョン :

権利関係 : 


\title{
Parametric instabilities of circularly polarized Alfvén waves in a relativistic electron-positron plasma
}

\author{
S. Matsukiyo* and T. Hada \\ Department of Earth System Science and Technology, Kyushu University, Fukuoka, 816-8580, Japan
}

(Received 11 June 2002; published 30 April 2003)

\begin{abstract}
Dispersion relation and nonlinear evolution of the parametric instabilities of circularly polarized Alfvén waves in a relativistic electron-positron plasma are investigated by theoretical and numerical approaches. In the nonrelativistic limit, when $2 \omega_{\mathrm{p}}^{2}>\omega_{0}^{2}$, the characteristics of the instabilities are similar to those in an electronion plasma, except that the modulational instability takes place only if $v_{\mathrm{s}}^{2}<\omega_{0}^{2} / k_{0}^{2}$, where $\omega_{\mathrm{p}}$ and $v_{\mathrm{s}}$ denote the plasma frequency and the acoustic speed, and $\omega_{0}, k_{0}$ indicate the frequency and the wave number of the parent wave. On the other hand, when $2 \omega_{\mathrm{p}}^{2}<\omega_{0}^{2}$, two new types of instabilities emerge between the parallel or antiparallel propagating Alfvén-like waves and the parallel propagating Langmuir-like wave. The weakly relativistic effect is discussed for all the instabilities. The one-dimensional full particle simulation and bicoherence analysis of the simulation result suggest that successive decay via the interaction between the parallel propagating Langmuir-like wave and antiparallel propagating Alfvén-like wave can efficiently generate a continuum of low frequency electromagnetic waves, which can interact with energetic particles.
\end{abstract}

DOI: 10.1103/PhysRevE.67.046406

PACS number(s): 52.35.Mw, 52.20.-j, 52.65.-y

\section{INTRODUCTION}

The particle acceleration and the magnetohydrodynamic (MHD) turbulence are inseparably related to each other. The parametric instability is thought to be a convincing candidate to produce the MHD turbulence. The resonance conditions for the three wave interaction are

$$
\omega_{3}=\omega_{1} \pm \omega_{2}, \quad k_{3}=k_{1} \pm k_{2},
$$

where $\left(\omega_{1}, \omega_{2}, \omega_{3}\right)$ and $\left(k_{1}, k_{2}, k_{3}\right)$ are the frequencies and wave numbers of the interacting waves. The evidence of the parametric instability of circularly polarized Alfvén waves in the earth's foreshock region has been reported (e.g., Spangler et al. [1,2]). Many authors have studied this instability by theoretical and numerical approaches. Galeev and Oraevskii [3], and Sagdeev and Galeev [4] first showed that the circularly polarized Alfvén waves are subject to parametric decay instability. Later, Goldstein [5] and Derby [6] derived the linear dispersion relation of the decay instability of circularly polarized Alfvén waves, as a four-wave interaction between a parent Alfvén wave and three (parallel and antiparallel electromagnetic waves and a parallel electrostatic wave) daughter waves. Weakly dispersive Alfvén waves are modulationally unstable if the wave number of density perturbation $k$ is much less than that of the parent wave $k_{0}$ (Mio et al. [7,8], Mjolhus [9], Sakai and Sonnerup [10]). In this case, three daughter waves all propagate parallel to the parent wave. The right- and left-hand polarized parent waves are unstable for $\beta>1$ and $\beta<1$, respectively, where $\beta=v_{\mathrm{si}}^{2} / v_{\mathrm{A}}^{2}$ is squared ratio of the ion acoustic to Alfvén speeds. A parametric study of the modulational instability for arbitrary magnitude of the dispersive effects is given by Longtin and

\footnotetext{
*Present address: Center for Interdisciplinary Plasma Science, Max-Planck-Institute for Extraterrestrial Physics, 85741 Garching, Germany.
}

Sonnerup [11]. Wong and Goldstein [12] and Terasawa et al. [13] compared the growth rates of the decay and modulational instabilities for dispersive Alfvén waves with an arbitrary amplitude. Wong and Goldstein [12] further pointed out the existence of the beat instability around $k \approx k_{0}$. In 1990s, the linear dispersion relations derived above are reinvestigated in detail by Jayanti and Hollweg [14], and Hollweg [15]. The effects of oblique propagation of daughter waves are also studied by several authors (Vinas and Goldstein $[16,17]$, and Ghosh et al. $[18,19])$.

On the other hand, Terasawa et al. [13] performed selfconsistent numerical simulations of the decay instability of circularly polarized Alfvén waves by utilizing a onedimensional hybrid simulation code. The numerical simulations can treat fully nonlinear dynamics of the system, while most of the past studies were concerned with the linear analyses. They investigated nonlinear evolution of the wave spectrum as well as heating of the plasma due to the ion acoustic wave. Machida et al. [20] also performed hybrid simulation study of the modulational instability and compared the results with those given by the DNLS (derivative nonlinear Schrödinger) equation (Mio et al. [7,8], Mjolhus [9], Spangler and Sheerin [21,22], Spangler [23-25], and Mjolhus and Wyller [26]). Hoshino and Goldstein [27] studied the nonlinear wave-wave couplings of the decay and modulational instabilities by means of the perturbation theory and the MHD simulations.

Most of the above studies have been discussed in the context of the solar wind plasma. However, the parametric decay processes of the Alfvén waves are important in high energy astrophysical environment such as the pulsar magnetosphere (Arons and Barnard [28]) and the neighborhood of extragalactic radio sources (Goncalves et al. [29]). These regions are considered to be filled with the electron-positron plasma. More than two decades ago, the parametric instabilities of circularly polarized waves in the electron-positron plasma are investigated by Sweeney and Stewart [30]. They discussed mainly the interaction between the high frequency 
electromagnetic parent wave and the sideband waves via the space charge fluctuations. Machabeli et al. [31] showed that almost parallel propagating high frequency transverse electromagnetic waves generated in the pulsar magnetosphere can be unstable through the density modulations parallel to the background magnetic field. Yoon and Ziebell [32] proposed a possible mechanism for the observed radiation from extragalactic radio jets based on the nonlinear interaction between the electron-positron jet and the intergalactic plasma.

Recently, the parametric instabilities of circularly polarized Alfvén waves in the relativistic electron-positron plasma are investigated by Muñoz and Gomberoff [33]. They obtained the linear dispersion relation and its numerical solutions assuming there is no space charge. In the electron-ion plasma, the frequency of the Alfvén wave should be smaller than the ion cyclotron frequency $\Omega_{\mathrm{i}}$. On the other hand, a characteristic frequency reflecting the space charge effect is the electron plasma frequency $\omega_{\mathrm{pe}}$. Since $\omega_{\mathrm{pe}}^{2} \gg \Omega_{\mathrm{i}}^{2}$ in usual solar wind plasma, ignoring the space charge effect is justified when we discuss the low frequency waves like the Alfvén wave. However, presence of the space charge is essential in the electron-positron plasma, since the plasma frequency $\sqrt{2} \omega_{\mathrm{p}}$ is often less than the cyclotron frequency $\Omega_{0}$. Therefore, it is important to study the parametric instabilities of the circularly polarized relativistic Alfvén waves including the effects of the space charge. In the electronpositron plasma the Alfvén and whistler waves have the identical dispersion and thus cannot be distinguished, so we will call them both the Alfvén waves in this paper.

The organization of this paper is as follows. In Sec. II, the basic equations are introduced and linearized. The obtained linear dispersion relation is discussed in Secs. III and IV for the nonrelativistic and the weakly relativistic cases, respectively. In Sec. V, the numerical simulation of parametric decay instability is performed by utilizing one-dimensional relativistic electromagnetic full particle code. Finally, the results are summarized and discussed in Sec. VI.

\section{FORMULATIONS AND BASIC ASSUMPTIONS}

Let us consider an electron-positron plasma, governed by the following equations.

$$
\begin{gathered}
\left(\frac{\partial}{\partial t}+\mathbf{v}_{\mathrm{j}} \cdot \boldsymbol{\nabla}\right)\left(\gamma_{\mathrm{j}} \mathbf{v}_{\mathrm{j}}\right)=\frac{q_{\mathrm{j}}}{m_{0}}\left(\mathbf{E}+\frac{\mathbf{v}_{\mathbf{j}} \times \mathbf{B}}{c}\right)-\frac{v_{\mathrm{s}}^{2}}{n_{0}} \boldsymbol{\nabla} n_{\mathrm{j}}, \\
\frac{\partial n_{\mathrm{j}}}{\partial t}=-\boldsymbol{\nabla} \cdot\left(n_{\mathrm{j}} v_{\mathrm{j}}\right), \\
\boldsymbol{\nabla} \cdot \mathbf{E}=4 \pi \rho, \\
\boldsymbol{\nabla} \times \mathbf{E}=-\frac{1}{c} \frac{\partial \mathbf{B}}{\partial t}, \\
\boldsymbol{\nabla} \times \mathbf{B}=\frac{4 \pi}{c} \mathbf{J}+\frac{1}{c} \frac{\partial \mathbf{E}}{\partial t},
\end{gathered}
$$

$$
\begin{gathered}
\mathbf{J}=\sum_{\mathrm{j}} q_{\mathrm{j}} n_{\mathrm{j}} \mathbf{v}_{\mathrm{j}}, \\
\rho=\sum_{\mathrm{j}} q_{\mathrm{j}} n_{\mathrm{j}},
\end{gathered}
$$

where $\mathbf{v}_{\mathrm{j}}=\left(u_{\mathrm{j}}, \mathbf{v}_{\perp j}\right)$ is the velocity, $\gamma_{\mathrm{j}}=1 / \sqrt{1-\mathbf{v}_{\mathrm{j}}^{2} / c^{2}}$ the Lorentz factor, $v_{\mathrm{s}}$ the acoustic speed, $n_{\mathrm{j}}$ the density, $q_{\mathrm{j}}$ the particle charge, $m_{0}$ the particle rest mass, $c$ the speed of light, and $\mathbf{E}$ and $\mathbf{B}$ are the electric and magnetic fields, respectively. The subscript $\mathrm{j}$ denotes particle species.

It is assumed that a circularly polarized Alfvén parent wave propagates parallel to the constant magnetic field $\mathbf{B}_{0}$ which is along the $x$ axis. Allowing both electrostatic and electromagnetic perturbations, we write

$$
\begin{gathered}
n_{\mathrm{j}}=n_{0}+\delta n_{\mathrm{j}}, \\
u_{\mathrm{j}}=\delta u_{\mathrm{j}}, \\
\mathbf{B}=\mathbf{B}_{0}+\mathbf{B}_{\mathrm{p}}+\delta \mathbf{B}, \\
\mathbf{v}_{\perp \mathrm{j}}=\mathbf{v}_{\mathrm{pj}}+\delta \mathbf{v}_{\mathrm{j}},
\end{gathered}
$$

where the subscript 0 represents the zeroth-order constant quantities, subscript $\mathrm{p}$ denotes the zeroth-order parent wave, and $\delta$ represents a small perturbation, respectively. These parent and perturbed quantities are further expressed as follows:

$$
\begin{gathered}
\mathbf{B}_{\mathrm{p}}=\frac{B_{\mathrm{p}}}{\sqrt{2}} \exp \left(-i \phi_{0}\right) \hat{\mathbf{e}}+\text { c.c. } \\
\delta \mathbf{B}=\frac{1}{\sqrt{2}}\left[\delta B_{+} \exp \left(-i \phi_{+}\right)+\delta B_{-} \exp \left(-i \phi_{-}\right)\right] \hat{\mathbf{e}}+\text { c.c. }
\end{gathered}
$$

$$
\delta n_{\mathrm{j}}=\frac{\delta n_{\mathrm{j}}}{2} \exp (-i \phi)+\text { c.c. }
$$

where $B_{\mathrm{p}}$ is real, $\phi_{0}=k_{0} x-\omega_{0} t, \phi_{ \pm}=k_{ \pm} x-\omega_{ \pm} t, \phi=k x$ $-\omega t,\left(k_{ \pm}, \omega_{ \pm}\right)=\left(k_{0} \pm k, \omega_{0} \pm \omega\right),\left(k_{0}, \omega_{0}\right)$ and $(k, \omega)$ are the wave number and frequency of parent wave and density perturbation, $\hat{\mathbf{e}}=(\hat{\mathbf{y}}-i \hat{\mathbf{z}}) / \sqrt{2}$ ( $\hat{\mathbf{y}}$ and $\hat{\mathbf{z}}$ are the unit vectors along respective directions), and c.c. denotes the complex conjugate. The parallel and perpendicular velocities are also written in a same manner.

For the zeroth order, the transverse velocity of each species is obtained as

$$
\frac{\mathrm{v}_{\mathrm{pj}}}{c}=-\frac{\omega_{0}}{c k_{0}} \frac{\Omega_{0 \mathrm{j}} \eta}{\gamma_{\mathrm{j}} \omega_{0}+\Omega_{0 \mathrm{j}}},
$$

where $\eta=B_{\mathrm{p}} / B_{0}$ and $\Omega_{0 \mathrm{j}}=q_{\mathrm{j}} B_{0} / m_{0} c$ is the nonrelativistic cyclotron frequency of species $\mathrm{j}$. The frequency of the parent wave, $\omega_{0}$, is given by the zeroth-order dispersion relation, 
(a)

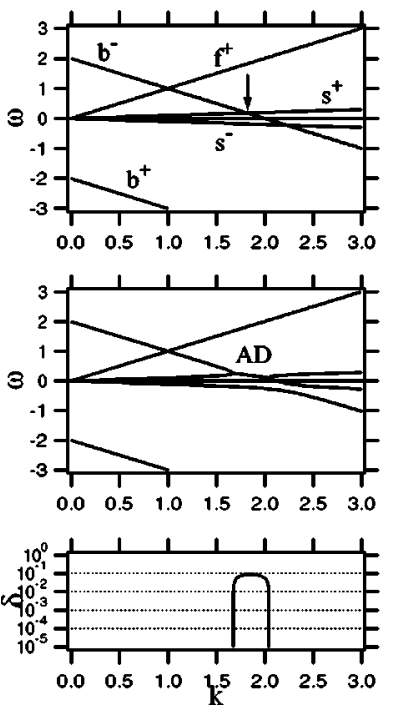

(b)
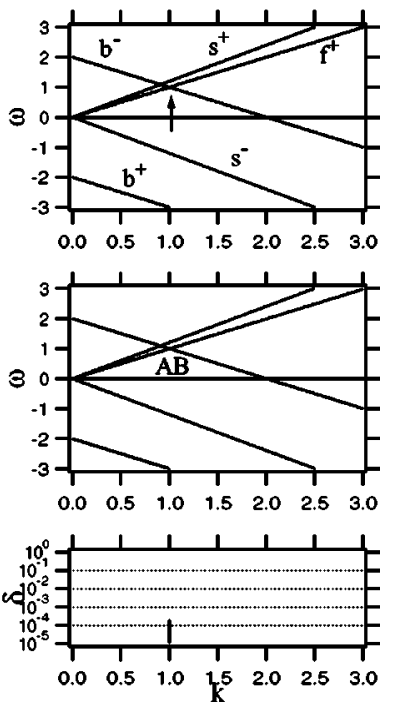

FIG. 1. Solutions of Eq. (35). The parameters are (a) $v_{\mathrm{s}}=0.1$, (b) $v_{\mathrm{s}}=1.2$, and $c^{2}=2.0$ is fixed. In each case, the top panel represents the frequency $\omega$ for $\eta=0$, the middle and the third panels show the real $\omega$ and imaginary $\delta$ frequencies for $\eta=0.1$ as a function of the wave number $k$.

$$
\frac{c^{2} k_{0}^{2}}{\omega_{0}^{2}}=1-\sum_{\mathrm{j}} \frac{\omega_{\mathrm{p}}^{2}}{\omega_{0}\left(\gamma_{\mathrm{j}} \omega_{0}+\Omega_{0 \mathrm{j}}\right)}
$$

Here, $\omega_{\mathrm{p}}$ is the plasma frequency. As noted by Muñoz and Gomberoff [33],

$$
\frac{c^{2} k_{0}^{2}}{\omega_{0}^{2}}=1-\sum_{\mathrm{j}} \frac{\omega_{\mathrm{p}}^{2}}{\omega_{0}\left(\omega_{0}+\Omega_{0 \mathrm{j}}\right)}\left[1-\frac{\eta^{2}}{2} \frac{\Omega_{0 \mathrm{j}}^{2}}{k_{0}^{2} c^{2}}\left(\frac{\omega_{0}}{\omega_{0}+\Omega_{0 \mathrm{j}}}\right)^{3}\right],
$$

provides a good approximation of Eq. (17) for weakly relativistic case. Equation (18) is derived from Eqs. (17) and (16) assuming $\left|\left(\omega_{0}+\Omega_{0 \mathrm{j}}\right) / \omega_{0}\right| \gg v_{\mathrm{pj}}^{2} / 2 c^{2}$. However, when the parent wave has a large amplitude and this inequality is not satisfied, Eqs. (17) and (16) must be used: with this respect, a part of the instability analysis by Muñoz and Gomberoff [33] appears to be misleading [cf. their Fig. 1(b)].

Hereafter, all of the variables are written in the unit of parent wave parameters for simplicity, i.e., the frequencies and wave numbers are normalized to $\omega_{0}$ and $k_{0}$, and the velocities to $\omega_{0} / k_{0}$, respectively. From Eqs. (2)-(15), to the first order of the perturbation amplitude, the following eigenvalue equations are obtained after some calculations.

$$
\begin{aligned}
& {\left[\omega_{+}\left(1+\frac{v_{\mathrm{p}}^{2}}{c^{2}}\right)+\Omega_{0}\right] \delta \mathrm{v}_{\mathrm{p}+}+\frac{v_{\mathrm{p}}^{2}}{2 c^{2}} \omega_{+} \delta \mathrm{v}_{\mathrm{p}-}^{*}} \\
& \quad-\frac{1}{2}\left[v_{\mathrm{p}}\left(1+\frac{v_{\mathrm{p}}^{2}}{2 c^{2}}\right)+\Omega_{0} \eta\right] \delta u_{\mathrm{p}}=-\frac{\omega_{+}}{k_{+}} \delta b_{+},
\end{aligned}
$$

$$
\begin{aligned}
& {\left[\omega_{-}\left(1+\frac{v_{\mathrm{p}}^{2}}{c^{2}}\right)+\Omega_{0}\right] \delta \mathrm{v}_{\mathrm{p}-}^{*}+\frac{v_{\mathrm{p}}^{2}}{2 c^{2}} \omega_{-} \delta \mathrm{v}_{\mathrm{p}+}} \\
& \quad-\frac{1}{2}\left[v_{\mathrm{p}}\left(1+\frac{v_{\mathrm{p}}^{2}}{2 c^{2}}\right)+\Omega_{0} \eta\right] \delta u_{\mathrm{p}}=-\frac{\omega_{-}}{k_{-}} \delta b_{-}^{*},
\end{aligned}
$$$$
\left.\omega_{+}\left(1+\frac{v_{\mathrm{e}}^{2}}{c^{2}}\right)-\Omega_{0}\right] \delta \mathrm{v}_{\mathrm{e}+}+\frac{v_{\mathrm{e}}^{2}}{2 c^{2}} \omega_{+} \delta \mathrm{v}_{\mathrm{e}-}^{*}
$$$$
-\frac{1}{2}\left[v_{\mathrm{e}}\left(1+\frac{v_{\mathrm{e}}^{2}}{2 c^{2}}\right)-\Omega_{0} \eta\right] \delta u_{\mathrm{e}}=\frac{\omega_{+}}{k_{+}} \delta b_{+},
$$$$
\left.\omega_{-}\left(1+\frac{v_{\mathrm{e}}^{2}}{c^{2}}\right)-\Omega_{0}\right] \delta \mathrm{v}_{\mathrm{e}-}^{*}+\frac{v_{\mathrm{e}}^{2}}{2 c^{2}} \omega_{-} \delta \mathrm{v}_{\mathrm{e}+}
$$$$
-\frac{1}{2}\left[v_{\mathrm{e}}\left(1+\frac{v_{\mathrm{e}}^{2}}{2 c^{2}}\right)-\Omega_{0} \eta\right] \delta u_{\mathrm{e}}=\frac{\omega_{-}}{k_{-}} \delta b_{-}^{*},
$$

$$
\begin{aligned}
{\left[\omega^{2}\left(1+\frac{v_{\mathrm{p}}^{2}}{2 c^{2}}\right)-k^{2} v_{\mathrm{s}}^{2}\right] \delta u_{\mathrm{p}}=} & \omega_{\mathrm{p}}^{2}\left(\delta u_{\mathrm{p}}-\delta u_{\mathrm{e}}\right) \\
& -\omega\left[v_{\mathrm{p}}\left(\delta b_{+}-\delta b_{-}^{*}\right)\right. \\
& \left.-\Omega_{0} \eta\left(\delta \mathrm{v}_{\mathrm{p}+}-\delta \mathrm{v}_{\mathrm{p}-}^{*}\right)\right]
\end{aligned}
$$

$$
\begin{aligned}
{\left[\omega^{2}\left(1+\frac{v_{\mathrm{e}}^{2}}{2 c^{2}}\right)-k^{2} v_{\mathrm{s}}^{2}\right] \delta u_{\mathrm{e}}=} & -\omega_{\mathrm{p}}^{2}\left(\delta u_{\mathrm{p}}-\delta u_{\mathrm{e}}\right) \\
& +\omega\left[v_{\mathrm{e}}\left(\delta b_{+}-\delta b_{-}^{*}\right)\right. \\
& \left.-\Omega_{0} \eta\left(\delta \mathrm{v}_{\mathrm{e}+}-\delta \mathrm{v}_{\mathrm{e}^{-}}^{*}\right)\right]
\end{aligned}
$$

$$
\begin{aligned}
\left(k_{+}^{2} c^{2}-\omega_{+}^{2}\right) \delta b_{+}= & \omega_{\mathrm{p}}^{2} k_{+}\left[\left(\delta \mathrm{v}_{\mathrm{p}+}-\delta \mathrm{v}_{\mathrm{e}+}\right)\right. \\
& \left.+\frac{k}{2 \omega}\left(v_{\mathrm{p}} \delta u_{\mathrm{p}}-v_{\mathrm{e}} \delta u_{\mathrm{e}}\right)\right], \\
\left(k_{-}^{2} c^{2}-\omega_{-}^{2}\right) \delta b_{-}^{*}= & \omega_{\mathrm{p}}^{2} k_{-}\left[\left(\delta \mathrm{v}_{\mathrm{p}-}^{*}-\delta \mathrm{v}_{\mathrm{e}-}^{*}\right)\right. \\
& \left.+\frac{k}{2 \omega}\left(v_{\mathrm{p}} \delta u_{\mathrm{p}}-v_{\mathrm{e}} \delta u_{\mathrm{e}}\right)\right],
\end{aligned}
$$

where $\Omega_{0}=e B_{0} / m_{0} c, \delta b_{ \pm}=e \delta B_{ \pm} / m_{0} c$, and the asterisk (*) implies the complex conjugate. In deriving the above equations, $v_{\mathrm{p}}^{2} / c^{2}, v_{\mathrm{e}}^{2} / c^{2} \ll 1$ is assumed. The above equations are identical to Eqs. (27)-(31) of Muñoz and Gomberoff [33], except that the terms $\pm \omega_{\mathrm{p}}^{2}\left(\delta u_{\mathrm{p}}-\delta u_{\mathrm{e}}\right)$, which originate from the finite space charge, are included in Eqs. (23) and 
(a)
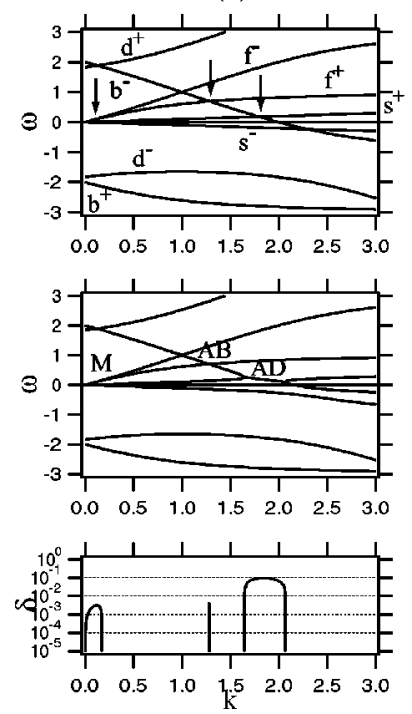

FIG. 2. Solutions of Eq. (34) with same format as Fig. 1. The parameters are (a) $v_{\mathrm{s}}=0.1$, (b) $v_{\mathrm{s}}=1.2$, and $c^{2}=2.0$ and $\Omega_{0}=2.0$ are fixed.

(24). The dispersion relation is given as a nontrivial solution (zero determinant) of Eqs. (19)-(26):

\section{STABILITY ANALYSES FOR NONRELATIVISTIC}

\section{PLASMA}

In the nonrelativistic limit $\left(v_{\mathrm{p}}^{2} / c^{2}, v_{\mathrm{e}}^{2} / c^{2} \rightarrow 0\right)$, the dispersion relation is obtained as follows:

$$
\begin{aligned}
& \omega_{\mathrm{p}}^{2}\left[2 S+A\left(C_{1}+C_{2}+C_{3}+C_{4}\right)\right]-S\left[S+A\left(C_{1}+C_{4}\right)\right] \\
& -A^{2}\left(C_{1} C_{4}-C_{2} C_{3}\right)=0, \\
& A=\frac{\Omega_{0}^{2} \eta^{2}}{2} \\
& S=\omega^{2}-k^{2} v_{\mathrm{s}}^{2} \\
& C_{1}=\frac{\omega}{1+\Omega_{0}}\left[\frac{\Omega_{0}^{2} k_{+}}{2 D_{+}}\left(\frac{\omega_{+}}{k_{+}\left(\omega_{+}+\Omega_{0}\right)}-\frac{1}{1+\Omega_{0}}\right)\right. \\
& \times\left(\frac{\Omega_{0}}{\omega_{+}+\Omega_{0}}-\frac{k}{\omega}\right)-\frac{\Omega_{0}^{2} k_{-}}{2 D_{-}}\left(\frac{\omega_{-}}{k_{-}\left(\omega_{-}+\Omega_{0}\right)}-\frac{1}{1+\Omega_{0}}\right) \\
& \left.\times\left(\frac{\Omega_{0}}{\omega_{-}+\Omega_{0}}-\frac{k}{\omega}\right)-\frac{\Omega_{0}\left(\omega_{-}-\omega_{+}\right)}{\left(\omega_{+}+\Omega_{0}\right)\left(\omega_{-}+\Omega_{0}\right)}\right], \\
& C_{2}=\frac{-\omega}{1-\Omega_{0}}\left[\frac{\Omega_{0}^{2} k_{+}}{2 D_{+}}\left(\frac{\omega_{+}}{k_{+}\left(\omega_{+}+\Omega_{0}\right)}-\frac{1}{1+\Omega_{0}}\right)\right. \\
& \times\left(\frac{\Omega_{0}}{\omega_{+}-\Omega_{0}}+\frac{k}{\omega}\right)-\frac{\Omega_{0}^{2} k_{-}}{2 D_{-}}\left(\frac{\omega_{-}}{k_{-}\left(\omega_{-}+\Omega_{0}\right)}-\frac{1}{1+\Omega_{0}}\right) \\
& \left.\times\left(\frac{\Omega_{0}}{\omega_{-}-\Omega_{0}}+\frac{k}{\omega}\right)\right]
\end{aligned}
$$

(a)

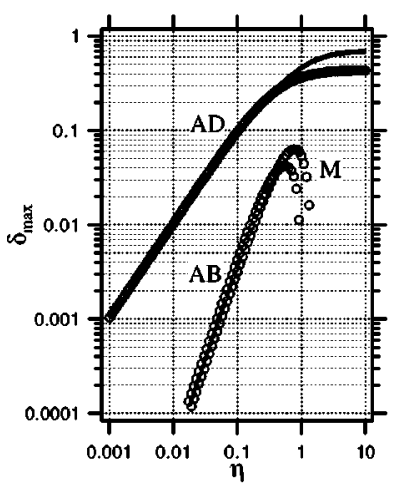

(b)

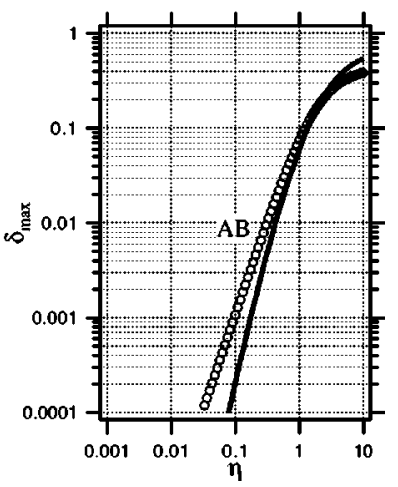

FIG. 3. Amplitude dependence of the maximum growth rate. The solid lines and the open circles correspond to the solutions of Eqs. (35) and (34), respectively. The parameters are (a) $v_{\mathrm{s}}=0.1$, (b) $v_{\mathrm{s}}=1.2$, and $c^{2}=\Omega_{0}=2.0$ are fixed.

$$
\begin{aligned}
C_{3}= & \frac{\omega}{1+\Omega_{0}}\left[\frac{\Omega_{0}^{2} k_{+}}{2 D_{+}}\left(\frac{\omega_{+}}{k_{+}\left(\omega_{+}-\Omega_{0}\right)}-\frac{1}{1-\Omega_{0}}\right)\right. \\
& \times\left(\frac{\Omega_{0}}{\omega_{+}+\Omega_{0}}-\frac{k}{\omega}\right)-\frac{\Omega_{0}^{2} k_{-}}{2 D_{-}}\left(\frac{\omega_{-}}{k_{-}\left(\omega_{-}-\Omega_{0}\right)}-\frac{1}{1-\Omega_{0}}\right) \\
& \left.\times\left(\frac{\Omega_{0}}{\omega_{-}+\Omega_{0}}-\frac{k}{\omega}\right)\right], \\
C_{4}= & \frac{-\omega}{1-\Omega_{0}}\left[\frac{\Omega_{0}^{2} k_{+}}{2 D_{+}}\left(\frac{\omega_{+}}{k_{+}\left(\omega_{+}-\Omega_{0}\right)}-\frac{1}{1-\Omega_{0}}\right)\right. \\
& \times\left(\frac{\Omega_{0}}{\omega_{+}-\Omega_{0}}+\frac{k}{\omega}\right)-\frac{\Omega_{0}^{2} k_{-}}{2 D_{-}}\left(\frac{\omega_{-}}{k_{-}\left(\omega_{-}-\Omega_{0}\right)}-\frac{1}{1-\Omega_{0}}\right) \\
& \left.\times\left(\frac{\Omega_{0}}{\omega_{-}-\Omega_{0}}+\frac{k}{\omega}\right)-\frac{\Omega_{0}\left(\omega_{-}-\omega_{+}\right)}{\left(\omega_{+}-\Omega_{0}\right)\left(\omega_{-}-\Omega_{0}\right)}\right]
\end{aligned}
$$

(a)
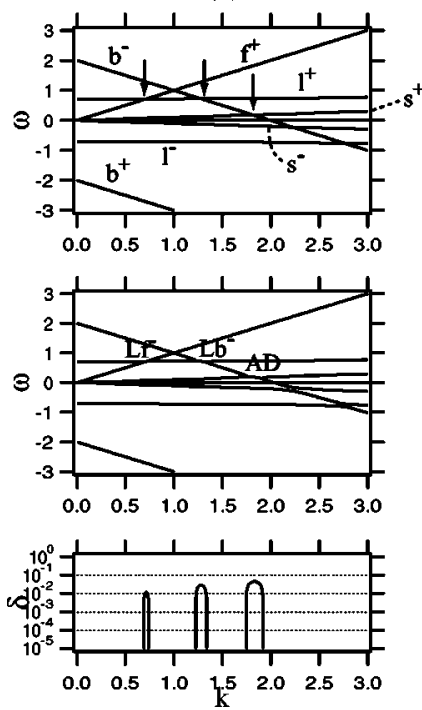

FIG. 4. Solutions of Eq. (38) with same format as Fig. 1. The parameters are (a) $\omega_{\mathrm{p}}=0.5, v_{\mathrm{s}}=0.1$, (b) $\omega_{\mathrm{p}}=1.0, v_{\mathrm{s}}=0.1$, and $\Omega_{0}$ $=2.0$ is fixed. (b)
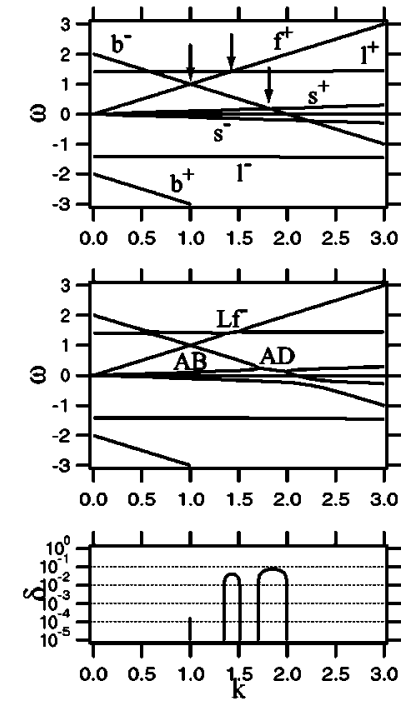


$$
D_{ \pm}=\left(k_{ \pm}^{2}-\frac{\omega_{ \pm}^{2}}{c^{2}}\right) v_{\mathrm{A}}^{2}+\frac{\Omega_{0}^{2} \omega_{ \pm}^{2}}{\omega_{ \pm}^{2}-\Omega_{0}^{2}},
$$

where $\quad v_{\mathrm{A}}^{2} / c^{2} \equiv \Omega_{0}^{2} / 2 \omega_{\mathrm{p}}^{2}=\Omega_{0}^{2} /\left(c^{2}-1\right)\left(\Omega_{0}^{2}-1\right) . \quad$ Equation (27) is appropriate for arbitrary amplitude of parent wave, and gives the same relation for right- $(\mathrm{R})$ and left-hand $(\mathrm{L})$ polarized waves. We discuss in detail the above dispersion relation for various parameter regime in the following sections.

Here, it may be convenient to define some labels used in following figures (Figs. 1-7).

Waves in the background plasma (for $\eta=0$ )

$f^{ \pm} \quad$ Parallel (forward) propagating Alfvén waves with $\left(\omega_{ \pm}, k_{ \pm}\right)$

$b^{ \pm} \quad$ Antiparallel (backward) propagating Alfvén waves with $\left(\omega_{ \pm}, k_{ \pm}\right)$

$s^{ \pm} \quad$ Parallel or antiparallel $(+$ or -$)$ propagating acoustic waves with $(\omega, k)$

$l^{ \pm} \quad$ Positive or negative $(+$ or - ) frequency Langmuir waves with $(\omega, k)$

$d^{ \pm} \quad$ High frequency electromagnetic waves with $\left(\omega_{ \pm}, k_{ \pm}\right)$

Types of the instability

AD Acoustic decay instability (main daughter waves are $s^{+}$and $b^{-}$)

AB Alfvén beat instability (main daughter waves are $f^{+}$ and $b^{-}$)

M Modulational instability (depends)

$\mathrm{Lb}^{-} \quad \mathrm{Lb}^{-}$instability (main daughter waves are $l^{+}$and $b^{-}$) [see text above Eq. (39)]

$\mathrm{Lf}^{-} \quad \mathrm{Lf}^{-}$instability (main daughter waves are $l^{+}$and $f^{-}$) [see text above Eq. (40)]

\section{A. Dispersion relation in a plasma without space charge}

$$
\left(\omega_{\mathrm{p}}^{2} \rightarrow \infty\right)
$$

When $\omega_{\mathrm{p}}^{2} \rightarrow \infty$, i.e., $\delta n_{\mathrm{e}}=\delta n_{\mathrm{p}}\left(\delta u_{\mathrm{e}}=\delta u_{\mathrm{p}}\right)$, Eq. (27) is reduced as

$$
2 S+A\left(C_{1}+C_{2}+C_{3}+C_{4}\right)=0 .
$$

After some calculations, this is rewritten as

$$
\begin{aligned}
& D_{+} D_{-} S-\frac{A \Omega_{0}^{2}}{1-\Omega_{0}^{2}}\left\{k_{+} D_{-}\left(\frac{\omega_{+}^{2}}{k_{+}\left(\omega_{+}^{2}-\Omega_{0}^{2}\right)}-\frac{1}{1-\Omega_{0}^{2}}\right)\right. \\
& \times\left[\frac{\omega\left(1+\omega_{+}\right) \Omega_{0}^{2}}{\left(\omega_{+}^{2}-\Omega_{0}^{2}\right)}+k\right]-k_{-} D_{+}\left(\frac{\omega_{-}^{2}}{k_{-}\left(\omega_{-}^{2}-\Omega_{0}^{2}\right)}-\frac{1}{1-\Omega_{0}^{2}}\right) \\
& \times\left[\frac{\omega\left(1+\omega_{-}\right) \Omega_{0}^{2}}{\left(\omega_{-}^{2}-\Omega_{0}^{2}\right)}+k\right] \\
& \left.+2 D_{+} D_{-} \frac{\omega^{2}\left(\omega_{+}+\omega_{-}+\omega_{+} \omega_{-}+\Omega_{0}^{2}\right)}{\left(\omega_{+}^{2}-\Omega_{0}^{2}\right)\left(\omega_{-}^{2}-\Omega_{0}^{2}\right)}\right\}=0
\end{aligned}
$$

(a)

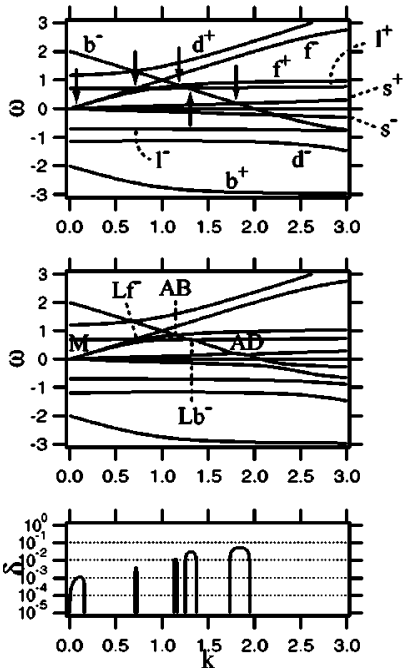

(b)
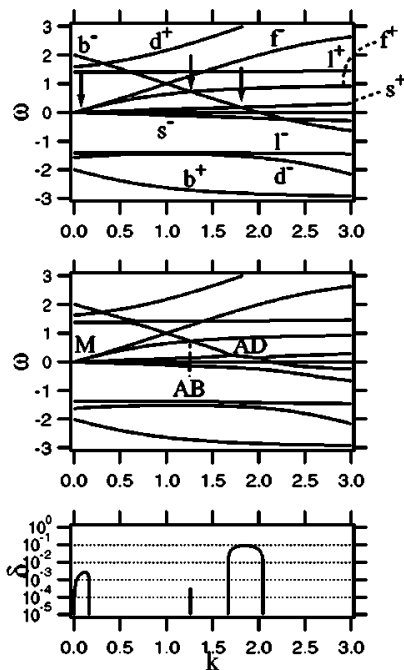

FIG. 5. Solutions of Eq. (27) with the same format as Fig. 1. The parameters are (a) $\omega_{\mathrm{p}}=0.5, v_{\mathrm{s}}=0.1$, (b) $\omega_{\mathrm{p}}=1.0, v_{\mathrm{s}}=0.1$, and $\Omega_{0}=2.0$ is fixed.

\section{Nondispersive case}

In the nondispersive limit $\left(\Omega_{0}^{2} \rightarrow \infty\right)$, Eq. (34) gives

$$
\begin{gathered}
\left(\omega^{2}-k^{2} v_{\mathrm{s}}^{2}\right)(\omega-k)(\omega+k+2)(\omega+k-2)-\frac{\eta^{2}}{2 v_{\mathrm{A}}^{2}}[(\omega+k \\
-2)\left(k-2 \omega-\omega^{2}\right)^{2}+(\omega+k+2)\left(k-2 \omega+\omega^{2}\right)^{2} \\
\left.-2 v_{\mathrm{A}}^{2} \omega^{2}(\omega-k)(\omega+k+2)(\omega+k-2)\right]=0,
\end{gathered}
$$

where $v_{\mathrm{A}}^{2}=c^{2} /\left(c^{2}-1\right)$ [the last equality holds since we let here the relativistic Alfvén velocity, $v_{\mathrm{A}}^{2} c^{2} /\left(c^{2}+v_{\mathrm{A}}^{2}\right)$ to be unity]. The above dispersion relation is the same as that obtained by Goldstein [5] and Derby [6] in the no displacement current limit $\left(v_{\mathrm{A}}^{2}=1\right.$ or $\left.c^{2} \rightarrow \infty\right)$ of the MHD. Numerical solutions of Eq. (35) with $c^{2}=2$ are shown in Fig. 1 for (a) $v_{\mathrm{s}}=0.1$ and (b) $v_{\mathrm{s}}=1.2$. The top panels correspond to the case with $\eta=0$ (no parent wave), so each labeled line represents the background plasma mode as defined above. The arrowed intersections show the interactions which will be unstable for finite $\eta$. The middle and bottom panels show the real and imaginary frequencies for $\eta=0.1$. The labels in the middle panels indicate the types of the instability which are also summarized above. This format is common in Figs. 1, 2, 4 , and 5. When $v_{\mathrm{s}}^{2}<1$ (a), usual decay instability, in which the parent wave decays into parallel propagating acousticlike wave $s^{+}$and antiparallel propagating Alfvén-like wave $b^{-}$ and parallel propagating electromagnetic wave $\left(\omega_{+}, k_{+}\right)$, takes place. Hereafter, we refer to this instability as the "acoustic" decay instability. When $\eta^{2} \ll 1, v_{\mathrm{A}} \sim O(1)$, and $0<1-v_{\mathrm{s}}^{2} \sim O(1)$, the maximum growth rate of this instability $\delta$ is obtained as

$$
\delta=\frac{\eta\left(1-v_{\mathrm{s}}\right)^{1 / 2}}{2 v_{\mathrm{A}} v_{\mathrm{s}}^{1 / 2}\left(1+v_{\mathrm{s}}\right)} .
$$


(a)

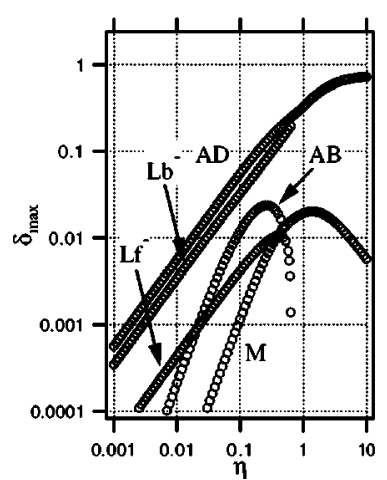

(b)

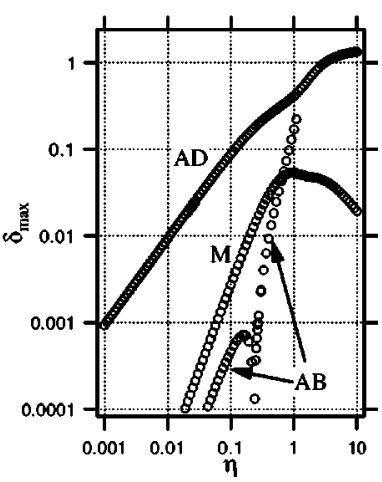

FIG. 6. Amplitude dependence of the maximum growth rate [solutions of Eq. (27)]. The parameters are (a) $\omega_{\mathrm{p}}=0.5$, (b) $\omega_{\mathrm{p}}$ $=1.0$, and $v_{\mathrm{s}}=0.1$ and $\Omega_{0}=2.0$ are fixed.

If there is no displacement current, $v_{\mathrm{A}}^{2}=1$, whereas the presence of the displacement current (finite $c$ ) let $v_{\mathrm{A}}^{2}$ be larger than unity, thus reducing the maximum growth rate, Eq. (36). The response of the plasma to the perturbation field is represented by the conduction current. According to the Maxwell's equation, when the wave is subluminous (Alfvénic), the conduction current is reduced due to the presence of the displacement current. As $v_{\mathrm{s}}^{2}$ exceeds unity (b), the acoustic decay instability is replaced by the beat instability in which the beating of the parent wave with the compressional wave $(\omega, k)$ generates the parallel $f^{+}$and antiparallel $b^{-}$propagating Alfvén-like waves (Jayanti and Hollweg [14]). We will call this as the Alfvén beat instability here. The maximum growth rate of this instability is derived as

$$
\delta=\frac{\eta^{3}}{4 \sqrt{2} v_{\mathrm{A}}^{3}\left(v_{\mathrm{s}}^{2}-1\right)^{3 / 2}} .
$$

As pointed out by Jayanti and Hollweg [14], the growth rate decreases rapidly as $v_{\mathrm{s}}^{2}$ increases since the density perturbation is hardly excited if $v_{\mathrm{s}}^{2}$ is large.

\section{Dispersive case}

If the dispersion effect is included, the modulational instability appears in addition to the acoustic decay and the Alfvén beat instabilities (cf. Wong and Goldstein [12]). In an electron-ion plasma, the right(left)-hand polarized parent wave is modulationally unstable for $v_{\mathrm{s}}^{2}>1(<1)$. On the other hand, in the electron-positron plasma, parent wave of either polarization is modulationally unstable when $v_{\mathrm{s}}^{2}<1$. This is because the lowest order dispersion, $\partial^{3} \omega_{0} / \partial k_{0}^{3}$, is always negative for the Alfvén waves in the electronpositron plasma. Therefore, the characteristics of the instabilities are similar to those in the electron-ion plasmas with left-hand polarized parent waves in which the lowest order dispersion, $\partial^{2} \omega_{0} / \partial k_{0}^{2}$, is negative. Figure 2 represents the numerical solutions of Eq. (34) for $\Omega_{0}=2.0$. Other parameters are the same as in Fig. 1. The modulational instability $k<1$ is seen only when $v_{\mathrm{s}}^{2}<1$. We note that, by including the dispersive effect, the Alfvén beat instability appears even (a)

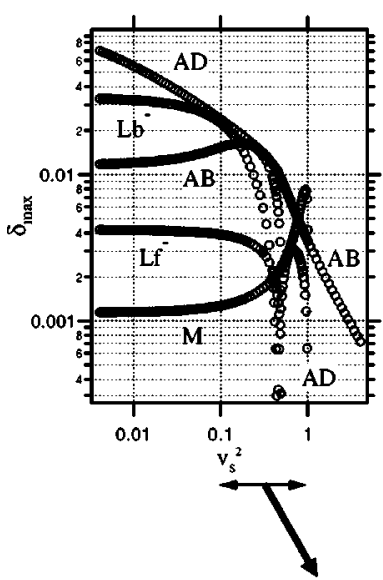

(b)

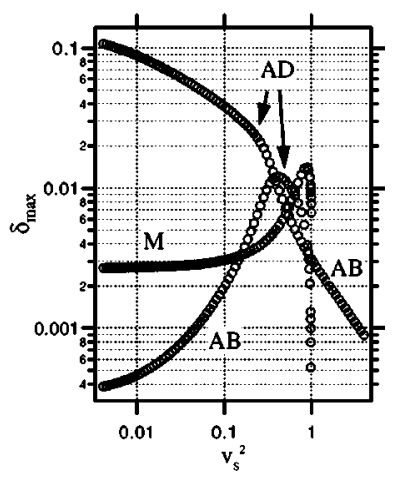

(c)

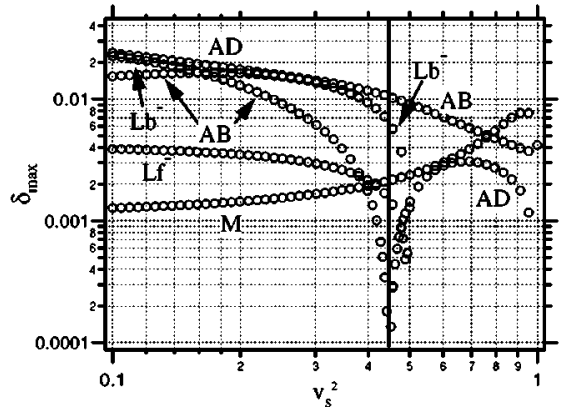

FIG. 7. Dependence of the maximum growth rate on $v_{\mathrm{s}}^{2}$ [solutions of Eq. (27)]. The parameters are (a) $\omega_{\mathrm{p}}=0.5$, (b) $\omega_{\mathrm{p}}=1.0$, and $\eta=0.1$ and $\Omega_{0}=2.0$ are fixed. The range $0.1<v_{\mathrm{s}}^{2}<1$ in (a) is expanded in (c).

when $v_{\mathrm{s}}^{2}<1$ (a), and the Alfvén beat instability growth rate is increased when $v_{\mathrm{s}}^{2}>1(\mathrm{~b})$. In order to see the amplitude dependence of each instability, their maximum growth rates are compared in Fig. 3. Each panel corresponds to (a) $v_{\mathrm{s}}=0.1$ and (b) $v_{\mathrm{s}}=1.2$. The solid lines and the open circles are obtained from Eqs. (35) and (34), respectively. Due to the dispersive effect, the acoustic decay (Alfvén beat) instability for $v_{\mathrm{s}}^{2}<1\left(v_{\mathrm{s}}^{2}>1\right)$ are enhanced at $\eta \leqq 0.1(\eta \leqq 1)$ and suppressed at $\eta \gtrsim 0.1(\eta \gtrsim 1)$. In addition, the modulational instability and the Alfvén beat instability for $v_{\mathrm{s}}=0.1$ are stabilized at sufficiently large $\eta$. These properties qualitatively agree with the results obtained for the electron-ion plasma (e.g., Wong and Goldstein [12], and Hollweg [15]). Note that the enhancement of the acoustic decay instability at $\eta \leq 0.1$ appears to be different from past studies since the normalization factors used here $\left(\omega_{0}\right.$ and $\left.k_{0}\right)$ are different from early works, e.g., Wong and Goldstein $[12]\left(\Omega_{0}\right.$ and $\left.v_{\mathrm{A}}\right)$.

\section{B. Dispersion relation in a plasma with finite space charge}

$$
\left(\omega_{\mathrm{p}}^{2} \ll \infty\right)
$$

When $\omega_{\mathrm{p}}^{2}$ is finite, we should fully solve Eq. (27). Then, the Langmuir wave emerges as a normal mode. Numerical solutions of Eq. (27) reveal that the Langmuir branch brings in drastic changes in the instabilities when $2 \omega_{\mathrm{p}}^{2}<\left(1+\Omega_{0}\right)^{2}$ (or $2 \omega_{\mathrm{p}}^{2}<1$ ), whereas characteristics of the instabilities remain qualitatively the same when $2 \omega_{\mathrm{p}}^{2}>\left(1+\Omega_{0}\right)^{2}$. Hence, only the former case is focused on here. 


\section{Nondispersive case}

If the dispersive effect is neglected, Eq. (27) is reduced as

$$
\begin{aligned}
& \left(\omega^{2}-k^{2} v_{\mathrm{s}}^{2}\right)\left(\omega^{2}-k^{2} v_{\mathrm{s}}^{2}-2 \omega_{\mathrm{p}}^{2}\right)(\omega-k)(\omega+k+2)(\omega+k-2) \\
& +\frac{\eta^{2}}{v_{\mathrm{A}}^{2}}\left\{\omega _ { \mathrm { p } } ^ { 2 } \left[(\omega+k-2)\left(k-2 \omega-\omega^{2}\right)^{2}\right.\right. \\
& +(\omega+k+2)\left(k-2 \omega+\omega^{2}\right)^{2} \\
& \left.-2 v_{\mathrm{A}}^{2} \omega^{2}(\omega-k)(\omega+k+2)(\omega+k-2)\right] \\
& \left.-S \Omega_{0}^{2}(\omega-k)^{2}(\omega+k)\right\} \\
& +\frac{\eta^{4}}{v_{\mathrm{A}}^{4}} \Omega_{0}^{2} \omega^{2}(\omega-k)\left[\omega^{2}-v_{\mathrm{A}}^{2}\left(\omega^{2}-k^{2}\right)\right]=0 .
\end{aligned}
$$

The numerical solutions of Eq. (38) are plotted in Fig. 4 in the same format as Fig. 1. Parameters used are (a) $\omega_{\mathrm{p}}=0.5$, $v_{\mathrm{s}}=0.1$, (b) $\omega_{\mathrm{p}}=1.0, v_{\mathrm{s}}=0.1$, with $\Omega_{0}=2.0$ for all the panels. The Langmuir waves $l^{ \pm}$are seen. In Fig. 4(a), two new types of interactions are observed in addition to the acoustic decay instability at $k \sim 1.8$, which is the only unstable mode when the space charge is absent [Fig. 1(a)]. The interaction at $k \sim 1.3$ is the decay type in which the antiparallel propagating Alfvén-like wave $b^{-}$interacts with the parallel propagating Langmuir-like $l^{+}$wave. We call this interaction the $\mathrm{Lb}^{-}$instability. The maximum growth rate of this instability is given by

$$
\begin{aligned}
& \delta=\frac{\eta}{2 v_{\mathrm{A}}}\left[\frac{(K-1)\left(4 \Omega_{0}^{2}-K^{2}\right)}{2(2-K)}\right]^{1 / 2}, \\
& K=\frac{2-\left[2 \omega_{\mathrm{p}}^{2}\left(1-v_{\mathrm{s}}^{2}\right)+4 v_{\mathrm{s}}^{2}\right]^{1 / 2}}{1-v_{\mathrm{s}}^{2}}
\end{aligned}
$$

where $K$ is the wave number at which the interaction takes place. This interaction is destabilized when $1<K(<2)$, i.e., $2 \omega_{\mathrm{p}}^{2}<1-v_{\mathrm{s}}^{2}$. On the other hand, at $k \sim 0.7$ another interaction between the parallel propagating Alfvén-like $f^{-}$and the Langmuir-like $l^{+}$waves is observed, which we call the $\mathrm{Lf}^{-}$ instability. The maximum growth rate is

$$
\delta=\frac{\eta \omega_{\mathrm{p}}}{\sqrt{2} v_{\mathrm{A}}\left(1-v_{\mathrm{s}}^{2}\right)^{1 / 2}} .
$$

In Fig. 4(b), the acoustic decay $(k \sim 1.8)$ and the $\mathrm{Lf}^{-}(k$ $\sim 1.4$ ) instabilities are still unstable. We should point out that $\mathrm{Lf}^{-}$instability may be in fact stable for $2 \omega_{\mathrm{p}}^{2}+k^{2} v_{\mathrm{s}}^{2}>1$, because the second term in the brackets in Eq. (38) $\left[-S \Omega_{0}^{2}(\omega-k)^{2}(\omega+k)\right]$ is underestimated in the dispersionless limit. The $\mathrm{Lb}^{-}$instability is replaced by the Alfvén beat instability $(k \sim 1.0)$ with the maximum growth rate

$$
\delta=\frac{\eta^{3} \omega_{\mathrm{p}}}{2 v_{\mathrm{A}}^{3}}\left[\frac{\omega_{\mathrm{p}}^{4}-\Omega_{0}^{2}\left(1-v_{\mathrm{s}}^{2}\right)^{2}}{\left(1-v_{\mathrm{s}}^{2}\right)^{3}\left(1-v_{\mathrm{s}}^{2}-2 \omega_{\mathrm{p}}^{2}\right)^{3}}\right]^{1 / 2} .
$$

The above expression gives (i) $v_{\mathrm{s}}^{2}>1, \omega_{\mathrm{p}}^{4}>\Omega_{0}^{2}\left(v_{\mathrm{s}}^{2}-1\right)^{2}$ or (ii) $0<1-v_{\mathrm{s}}^{2}<2 \omega_{\mathrm{p}}^{2}<2 \Omega_{0}\left(1-v_{\mathrm{s}}^{2}\right)$ as the conditions of the instability. However, as $v_{\mathrm{s}}^{2}$ is increased in (i), the growth rate is decreased because of the high thermal pressure, while as $\omega_{\mathrm{p}}^{2}$ is increased in (ii), the growth rate is reduced due to strong electrostatic restoring force.

\section{Dispersive case}

Numerical solutions of Eq. (27) are shown in Fig. 5, using the same parameters as in Fig. 4. The modulational instability (at $k \ll 1$ ) and the Alfvén beat instability [(a) at $k \sim 1.2$, (b) at $k \sim 1.3$ ] appear, as in the no space charge limit when we compared Figs. 1 and 2 . The Lf $^{-}$instability is stabilized in Fig. 5(b). The growth rates of the instabilities are compared in Fig. 6 as a function of $\eta$. The parameters are (a) $\omega_{\mathrm{p}}=0.5$, (b) $\omega_{\mathrm{p}}=1.0$, and $v_{\mathrm{s}}=0.1$ and $\Omega_{0}=2.0$ are fixed. In Fig. 6(a), the acoustic decay and the $\mathrm{Lb}^{-}$instabilities have the largest and the second largest growth rates, while the modulational instability has the smallest growth rate for any $\eta$. The $\mathrm{Lf}^{-}$instability has a larger growth rate than that of the Alfvén beat instability at small $\eta$. The magnitude of the growth rate of the $\mathrm{Lb}^{-}$instability is of the same order of that of the acoustic decay instability. In Fig. 6(b), the acoustic decay instability is again the most dominant. Dependence of the maximum growth rates on $v_{\mathrm{s}}^{2}$ is shown in Fig. 7 for $\Omega_{0}$ $=2.0, \eta=0.1$, and (a) $\omega_{\mathrm{p}}=0.5$ and (b) $\omega_{\mathrm{p}}=1.0$. When $\omega_{\mathrm{p}}$ $=1.0[$ Fig. 7(b)], three types of instabilities are seen. The acoustic decay instability is dominant for $v_{\mathrm{s}}^{2} \lesssim 0.65$, especially if $v_{\mathrm{s}}^{2} \lesssim 0.1$ it is much more dominant than the other two instabilities. The maximum growth rates change rapidly around $v_{\mathrm{s}}^{2} \sim 0.4$, where the acoustic decay and the Alfvén beat instabilities degenerate. When $0.65 \leqq v_{\mathrm{s}}^{2} \lessgtr 1$, the modulational instability is the most dominant, and when $v_{\mathrm{s}}^{2}>1$ only the Alfvén beat instability is unstable. The situation is more complicated when $\omega_{\mathrm{p}}=0.5[$ Fig. $7(\mathrm{a})]$. Figure $7(\mathrm{c})$ is an expansion of (a). While the acoustic decay instability is the most dominant when $v_{\mathrm{s}}^{2} \lesssim 0.45$, the $\mathrm{Lb}^{-}$instability also has the same order of magnitude of maximum growth rate. The Alfvén beat instability becomes the most dominant for 0.45 $\lesssim v_{\mathrm{s}}^{2} \lessgtr 0.75$, and at the same time the growth rates of the $\mathrm{Lb}^{-}$and the acoustic decay instabilities suddenly decrease. The jump of the growth rates of the acoustic decay and the Alfvén beat instabilities at $v_{\mathrm{s}}^{2} \sim 0.45$ are again due to the degeneracy of roots. For $0.75 \lesssim v_{\mathrm{s}}^{2} \lesssim 1$ the modulational instability is the most dominant, and when $v_{\mathrm{s}}^{2}>1$, only the Alfvén beat instability is unstable.

\section{STABILITY ANALYSES FOR RELATIVISTIC PLASMA}

The relativistic correction of the dispersion relation, Eq. (27), is discussed here. We consider a weakly relativistic case, i.e., only terms up to the second order of the parent wave amplitude is retained ( $\eta \ll 1$ is assumed). After some calculations, the following dispersion relation is obtained.

$$
\begin{gathered}
\omega_{\mathrm{p}}^{2}\left[S_{\mathrm{p}}+S_{\mathrm{e}}+A\left(C_{1}+C_{2}+C_{3}+C_{4}\right)\right] \\
-\left[S_{\mathrm{p}} S_{\mathrm{e}}+A S_{0}\left(C_{1}+C_{4}\right)\right]=0,
\end{gathered}
$$


where

$$
\begin{gathered}
S_{\mathrm{e}}=\omega^{2}\left[1+\frac{\Omega_{0}^{2} \eta^{2}}{c^{2}\left(1-\Omega_{0}\right)^{2}}\right]-k^{2} v_{\mathrm{s}}^{2}, \\
S_{\mathrm{p}}=\omega^{2}\left[1+\frac{\Omega_{0}^{2} \eta^{2}}{c^{2}\left(1+\Omega_{0}\right)^{2}}\right]-k^{2} v_{\mathrm{s}}^{2}, \\
S_{0}=\omega^{2}-k^{2} v_{\mathrm{s}}^{2} .
\end{gathered}
$$

Equation (42) is analogous to Eq. (27), but the expression of $S$ is modified by the relativistic effect, and the third term in Eq. (27) proportional to $A^{2}$ is omitted due to the weakly relativistic assumption above. We note that, ignoring the displacement current is quantitatively equivalent to ignoring the relativistic effect, since a factor of $1 / c^{2}\left(\omega_{0}^{2} / k_{0}^{2} c^{2}\right)$ is included in the relativistic correction terms of $S_{e}$ and $S_{p}$ [Eqs. (43) and (44)]. Therefore, the displacement current is again included here.

Let us briefly discuss the qualitative differences between Eqs. (42) and (27). In the nondispersive limit, Eq. (42) is reduced as

$$
\begin{aligned}
& g^{4}\left(\omega^{2}-\frac{k^{2} v_{\mathrm{s}}^{2}}{g^{2}}\right)\left(\omega^{2}-\frac{k^{2} v_{\mathrm{s}}^{2}+2 \omega_{\mathrm{p}}^{2}}{g^{2}}\right)(\omega-k)(\omega+k+2) \\
& \quad \times(\omega+k-2)+\frac{\eta^{2}}{v_{\mathrm{A}}^{2}}\left\{\omega _ { \mathrm { p } } ^ { 2 } \left[(\omega+k-2)\left(k-2 \omega-\omega^{2}\right)^{2}\right.\right. \\
& +(\omega+k+2)\left(k-2 \omega+\omega^{2}\right)^{2} \\
& -2 v_{\mathrm{A}}^{2} \omega^{2}(\omega-k)(\omega+k+2) \\
& \left.\times(\omega+k-2)]-g^{2} \Omega_{0}^{2}\left(\omega^{2}-\frac{k^{2} v_{\mathrm{s}}^{2}}{g^{2}}\right)(\omega-k)^{2}(\omega+k)\right\},
\end{aligned}
$$

where $g^{2}=1+\eta^{2} / c^{2}$. If $v_{\mathrm{s}}^{2} / g^{2}, \omega_{\mathrm{p}}^{2} / g^{2}$, and $\eta^{2} / g^{2}$ are, respectively, replaced by $v_{\mathrm{s}}^{2}, \omega_{\mathrm{p}}^{2}$, and $\eta^{2}$ in Eq. (46), the equation becomes identical to Eq. (38). Therefore, inclusion of the relativity effectively decreases the acoustic speed, the plasma frequency, and the parent wave amplitude. As a result, the maximum growth rate of the acoustic decay instability is less (greater) than Eq. (36) for $v_{\mathrm{s}}<1 / 3\left(v_{\mathrm{s}}>1 / 3\right)$, and those of the $\mathrm{Lb}^{-}$and the $\mathrm{Lf}^{-}$instabilities are less than Eqs. (39) and (40), respectively. In order to compare the Alfvén beat instability with the nonrelativistic case [Eq. (41)], the contribution from the fourth-order term of $\eta$ should be included in Eqs. (42) and (46). In the dispersive case, on the other hand, we numerically confirmed that the modulational instability is suppressed by the relativistic effect.

\section{NUMERICAL SIMULATION}

In order to investigate time evolution of the acoustic decay and the $\mathrm{Lb}^{-}$instabilities, which are dominant when
TABLE I. Physical parameters for simulation.

\begin{tabular}{lccc}
\hline \hline \multicolumn{2}{c}{ Parameters of the parent wave } & \multicolumn{2}{c}{ Plasma } \\
\hline$\eta$ & 0.1 & $\omega_{\mathrm{p}}$ & 0.4 \\
$\omega_{0}$ & 1.93762 & $\Omega_{0}$ & 4.0 \\
Mode number & 32 & $v_{\text {th }} / c$ & 0.04 \\
\hline \hline
\end{tabular}

$2 \omega_{\mathrm{p}}^{2}<1$, numerical simulation is performed by using relativistic electromagnetic full particle code, in which equation of motion for individual electrons and positrons and the Maxwell's equations are simultaneously solved in a selfconsistent manner. The simulation has only one spatial dimension (the $x$ direction), while all the three velocity dimensions are retained. In the simulation run below, we let the background dc magnetic field be aligned with the $x$ axis, so that it is assumed that all the waves we are dealing with are either parallel or antiparallel propagating with respect to the dc magnetic field.

Again, let us explain here some labels used in remaining figures.

$P \quad$ Parent wave

$R_{\mathrm{AD}} R$ mode wave generated by the acoustic decay instability

$R_{\text {Lb }} \quad R$ mode wave generated by the $\mathrm{Lb}^{-}$instability

$R_{1} \quad R$ mode wave generated in the nonlinear stage

$R_{2} \quad R$ mode wave generated in the nonlinear stage

$E_{\mathrm{AD}}$ Electrostatic wave generated by the acoustic decay instability

$E_{\mathrm{Lb}} \quad$ Electrostatic wave generated by the $\mathrm{Lb}^{-}$instability

$E_{1} \quad$ Electrostatic wave generated in the nonlinear stage

$E_{2} \quad$ Electrostatic wave generated in the nonlinear stage

\section{A. Parameters and initial conditions}

The parallel propagating monochromatic right-hand circularly polarized Alfvén wave, represented by Eq. (13), is introduced as a parent wave. The initial particle velocities are given by Eq. (16) with Eq. (17). The total number of the spatial grids in the simulation system is 16384 . The number of particles in each cell is 50 for both electrons and positrons. The system length is $256.0\left(=40.96 \mathrm{c} / \omega_{\mathrm{p}}\right)$ and the time step is $0.00625\left(\approx 0.012 \omega_{0}^{-1}\right)$. The physical parameters used are shown in Table I.

\section{B. Simulation results}

Figure 8 shows the $\omega-k$ power spectrum of $B_{\mathrm{z}}$ and $E_{\mathrm{x}}$ components in the linear stage $\left(198.4 \leqslant \omega_{0} t \leqslant 396.0\right)$. The gray scale denotes the wave power in the logarithmic scale. As expected from the linear theory, the parent wave $(P)$ decays into two antiparallel propagating electromagnetic waves $\left(R_{\mathrm{AD}}\right.$ and $\left.R_{\mathrm{Lb}}\right)$ and two parallel propagating electrostatic waves $\left(E_{\mathrm{AD}}\right.$ and $\left.E_{\mathrm{Lb}}\right)$. A small peak at $E_{1}$ is produced via beating of either $\mathrm{R}_{\mathrm{AD}}$ and $R_{\mathrm{Lb}}$, or $E_{\mathrm{AD}}$ and $E_{\mathrm{Lb}}$. This point will be further analyzed later. We should comment here on the strong vertical bars. The data used here is periodic in space but nonperiodic in time. Especially in the linear stage, the wave amplitude grows exponentially in time. Therefore, the Fourier transformation to such data gives broadband 
(a)
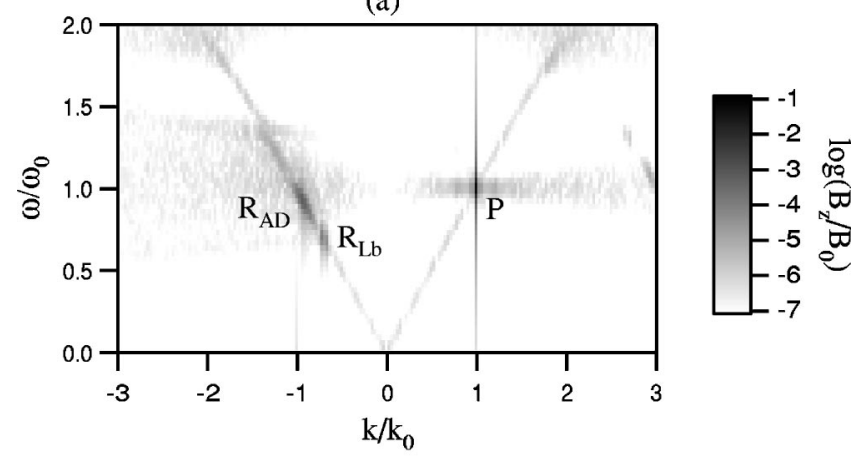

(b)
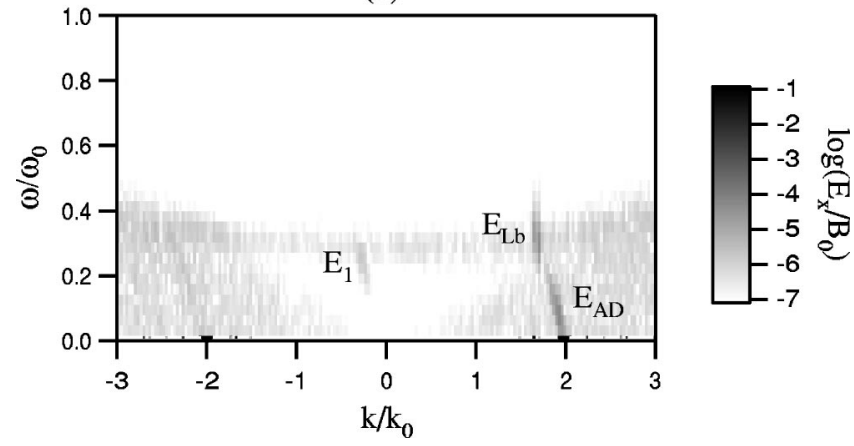

FIG. 8. $\omega-k$ power spectra obtained for the period of 198.4 $\leqslant \omega_{0} t \leqslant 396.0$.

noise. One may avoid this artifact by using, for instance, the wavelet transformation (Dudok de Wit and Krasnosel'skikh [34]).

The time evolution of the $k$ spectra is represented in Fig. 9. The top panel shows power of the electromagnetic wave which consists of parallel propagating $R$ mode waves and antiparallel propagating L mode waves (we call them the $R^{+}$ and $L^{-}$waves, respectively). In the middle panel, the electromagnetic wave including $R^{-}$and $L^{+}$waves is shown. The bottom panel is the power of $E_{\mathrm{x}}$. In the early stage $\left(\omega_{0} t\right.$ $\sim 200$ ), only the waves expected by the linear theory are observed $\left(R_{\mathrm{AD}}, R_{\mathrm{Lb}}, E_{\mathrm{AD}}\right.$, and $\left.E_{\mathrm{Lb}}\right)$. On the other hand, at $\omega_{0} t \sim 400, E_{1}$ is generated, and later $R_{1}, R_{2}$, and $E_{2}$ are excited one after another. One can tell from the resonance condition, Eq. (1), that either coupling between $R_{\mathrm{AD}}$ and $R_{\mathrm{Lb}}$, or between $E_{\mathrm{AD}}$ and $E_{\mathrm{Lb}}$ is producing $E_{1}$. In general, however, it is difficult to identify the origin of each wave when many wave modes coexist. For instance, there are a few possibilities to produce the wave modes $R_{2}$ and $E_{2}$. One possibility is that both $R_{2}$ and $E_{2}$ are generated by the interaction of $E_{1}$ and other wave modes. Another possibility is that $R_{2}$ is produced by the coupling between $E_{1}$ and $R_{\mathrm{Lb}}$, and $E_{2}$ is generated from the coupling between $R_{2}$ and the parent wave $P$. Therefore, in order to determine the origin of waves produced by two wave coupling process, we make use of the bicoherence analysis, as described below.

\section{Bicoherence analysis}

The bicoherence analysis is a valuable method to evaluate the third-order correlation (see Dudok de Wit and
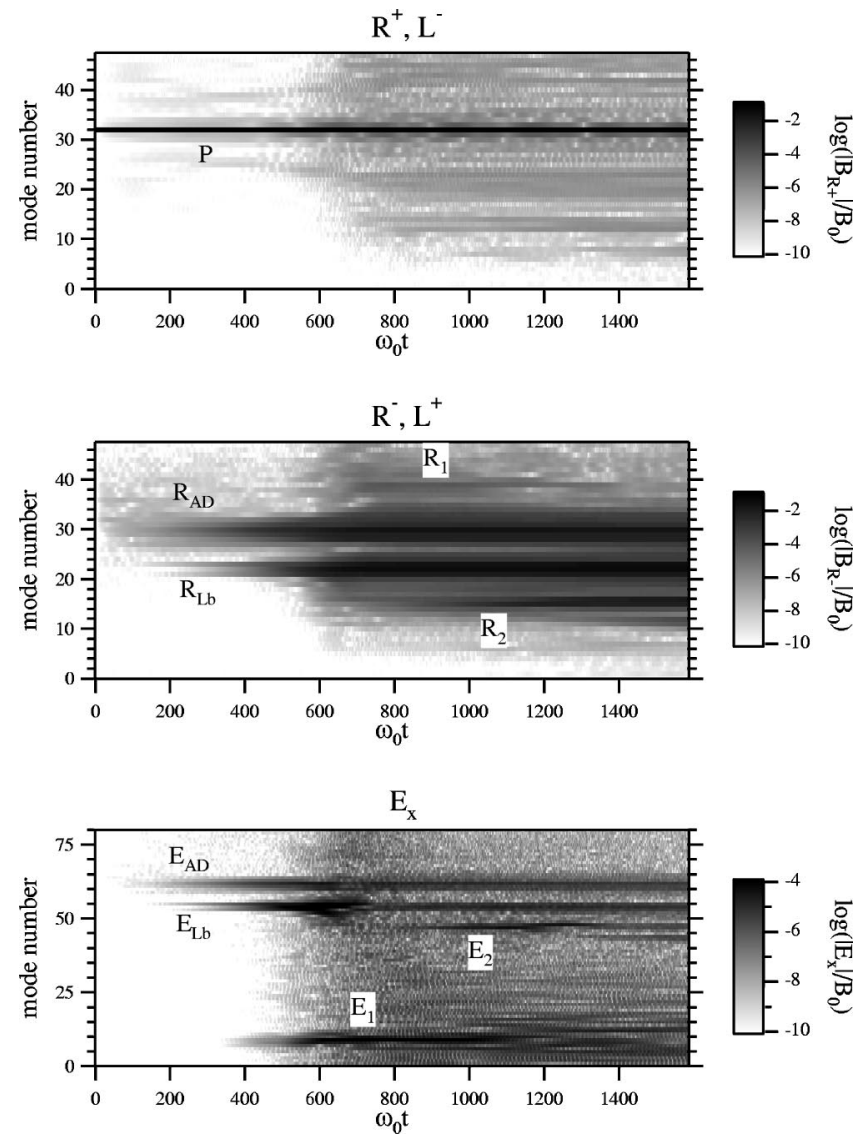

FIG. 9. Time evolution of $k$ spectra.

Krasnosel'skikh [34], and references therein) in a given time series data. The (cross)bispectrum is defined as

$$
B\left(m_{1}, m_{2}\right)=\left\langle X\left(m_{1}\right) Y\left(m_{2}\right) Z^{*}\left(m_{1}+m_{2}\right)\right\rangle,
$$

where $X, Y$, and $Z$ are the Fourier transform of a given time series, $m_{1}$ and $m_{2}$ are the mode numbers (or the frequencies), the asterisk denotes the complex conjugate, and the bracket denotes the ensemble average. The bicoherence is obtained by normalizing the bispectrum,

$$
b^{2}\left(m_{1}, m_{2}\right)=\frac{\left|B\left(m_{1}, m_{2}\right)\right|^{2}}{\left\langle\left|X\left(m_{1}\right) Y\left(m_{2}\right) Z^{*}\left(m_{1}+m_{2}\right)\right|\right\rangle^{2}} .
$$

The finite values of $b^{2}\left(m_{1}, m_{2}\right)$ imply the existence of a phase coherence (the nonlinear interaction) between the wave modes at $m_{1}, m_{2}$, and $m_{1}+m_{2}$.

Using the dataset obtained by the numerical simulation, we have performed the bicoherence analysis. Figure 10 shows the result obtained for the period $198.4 \leqslant \omega_{0} t$ $\leqslant 396.0$. The upper left panel denotes the bicoherence between $B_{\mathrm{z}}\left(m_{1}\right), B_{\mathrm{z}}\left(m_{2}\right)$, and $E_{\mathrm{x}}^{*}\left(m_{1}+m_{2}\right)$. A strong interaction occurs between mode numbers $m_{1}=32$ and $m_{2}=27$ $\sim 31$. Since $m_{1}$ and $m_{2}$ correspond to the mode numbers of the parent wave $P$ and $R_{\mathrm{AD}}, m_{1}+m_{2}=59 \sim 63$ corresponds to the mode number of $E_{\mathrm{AD}}$. This interaction, therefore, is confirmed to be the acoustic decay instability. Similarly, the interaction between $m_{1}=32$ and $m_{2}=21 \sim 23$ is shown to be 

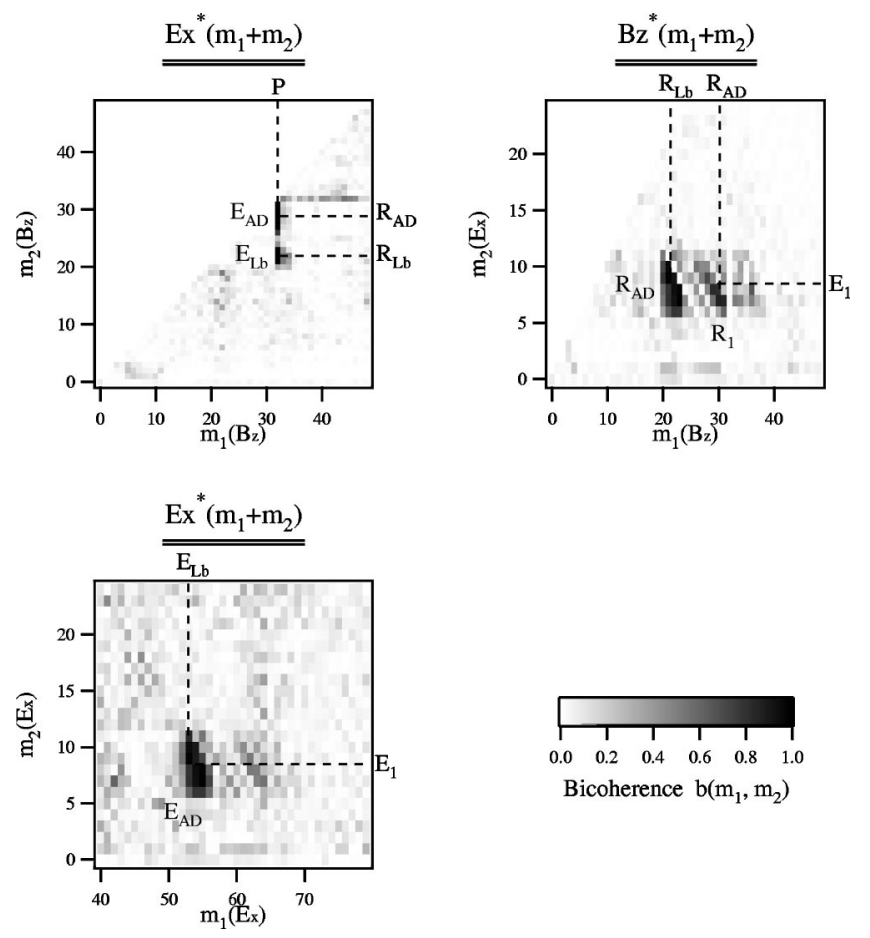

FIG. 10. Bicoherence obtained for the period of $198.4 \leqslant \omega_{0} t$ \$396.0. (i) The upper left panel: the bicoherence between $B_{z}\left(m_{1}\right), B_{z}\left(m_{2}\right)$, and $E_{x}^{*}\left(m_{1}+m_{2}\right)$. (ii) The upper right panel: the bicoherence between $B_{z}\left(m_{1}\right), E_{x}\left(m_{2}\right)$ and $B_{z}^{*}\left(m_{1}+m_{2}\right)$. (iii) The lower panel: the bicoherence between $E_{x}\left(m_{1}\right), E_{x}\left(m_{2}\right)$ and $E_{x}^{*}\left(m_{1}\right.$ $\left.+m_{2}\right)$. Here, $m_{1}$ and $m_{2}$ denote mode numbers of the corresponding wave component.

the $\mathrm{Lb}^{-}$instability. In the upper right panel, the bicoherence between $B_{\mathrm{z}}\left(m_{1}\right), E_{\mathrm{x}}\left(m_{2}\right)$, and $B_{\mathrm{z}}^{*}\left(m_{1}+m_{2}\right)$ is presented. The strongest peak at $m_{1}=21 \sim 23$ and $m_{2}=7 \sim 10$ corresponds to the interaction between $R_{\mathrm{AD}}, R_{\mathrm{Lb}}$, and $E_{1}$. The interaction between $E_{\mathrm{AD}}, E_{\mathrm{Lb}}$, and $E_{1}$ appears in the lower panel, which is the bicoherence between $E_{\mathrm{x}}\left(m_{1}\right), E_{\mathrm{x}}\left(m_{2}\right)$, and $E_{\mathrm{x}}^{*}\left(m_{1}+m_{2}\right)$. There exists an unexpected peak in the upper right panel at $m_{1}=29 \sim 31$ and $m_{2}=7 \sim 8$. This implies that the $R_{1}$, whose amplitude is still small at this time, is going to be produced via the beating of $E_{1}$ and $R_{\mathrm{AD}}$.

Figure 11 shows the bicoherence spectrum obtained at later time period $992.0 \leqslant \omega_{0} t \leqslant 1189.6$. In this period, a variety of wave modes are generated (cf. Fig. 9). Here, we pay particular attention to the generation mechanism of $R_{2}$ and $E_{2}$. First, the strong interaction between $R_{2}, E_{2}$, and $P$ is recognized in the upper left panel. At the same time, if $R_{2}$ is produced via the beating of $E_{1}$ and $R_{\mathrm{Lb}}$ as discussed in the preceding section, a peak should appear around the marked point in the upper right panel. The interaction is seen, but the peak intensity is weak compared with that of the interaction between $R_{2}, E_{2}$, and $P$. Similarly, it is found in the lower panel that the interaction between $E_{1}, E_{\mathrm{Lb}}$, and $E_{2}$ is weak. Hence, we conclude that generation of $R_{2}$ and $E_{2}$ is not via coupling between $E_{1}$ and other wave modes, but is due to decay (second stage) of the parent wave $\left(P \rightarrow R_{2}+E_{2}\right)$ in a plasma already thermalized by the first stage of the decay.
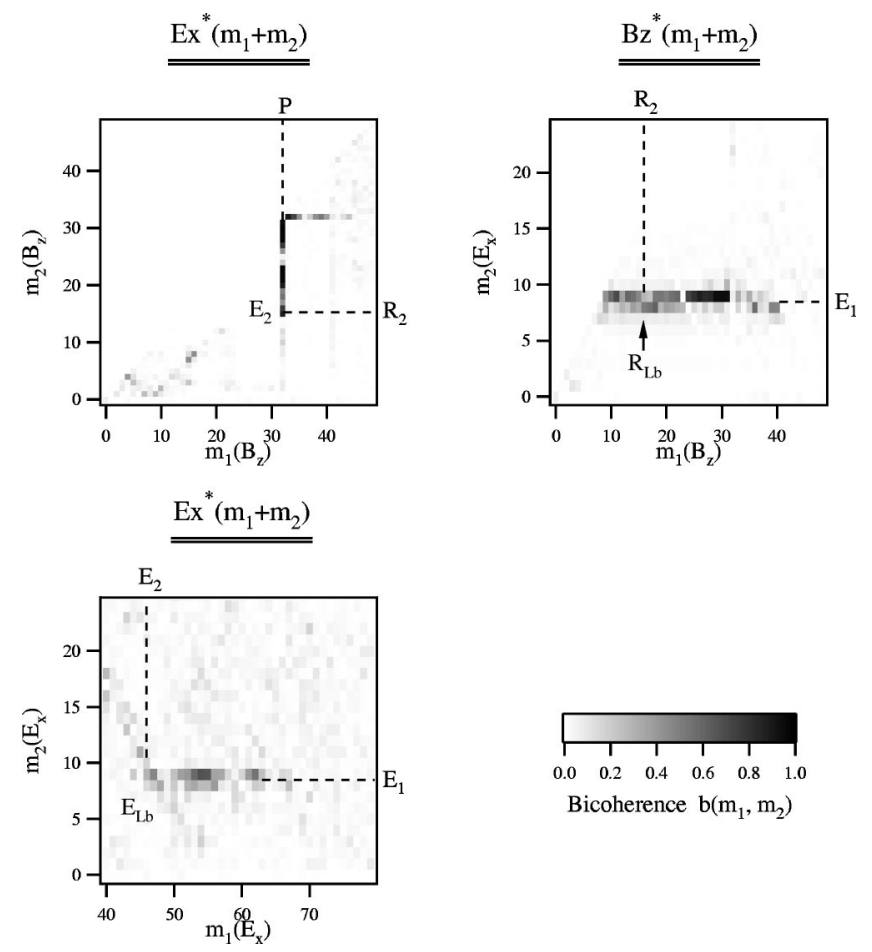

FIG. 11. Bicoherence obtained for the period of $992.0 \leqslant \omega_{0} t$ $\leqslant 1189.6$ with same format as Fig. 10 .

\section{Second decay instability}

The time evolution of the parallel drift $\left(k_{0} v_{\mathrm{d}} / \omega_{0}\right)$ and thermal $\left(k_{0} v_{\text {th }} / \omega_{0}\right)$ velocities of electrons are shown in Fig. 12(a), and the electron parallel distribution functions at $\omega_{0} t$ $=387.5$, 581.3, and 775.0 are presented in Fig. 12(b). The rapid increase of $k_{0} v_{\mathrm{d}} / \omega_{0}$ and $k_{0} v_{\mathrm{th}} / \omega_{0}$ at $380<\omega_{0} t<780$ is found in (a). This is explained as follows. In (b), the broadening of the distribution function in $0<k_{0} v_{\mathrm{x}} / \omega_{0}<0.1$ at $\omega_{0} t=387.5$ and 581.3 is caused by the resonant trapping due to the acoustic waves driven by the first acoustic decay instability. In addition, the clear plateau in $0.16<k_{0} v_{\mathrm{x}} / \omega_{0}$ $<0.3$ at $\omega_{0} t=581.3$ is produced through the resonant interaction with the Langmuir waves excited by the first $\mathrm{Lb}^{-}$ instability. As a result of these heating and acceleration, the dispersion relation of the background plasma is modified. The phase velocity of $E_{2}$ is estimated as $0.34 \sim 0.36$, and it is approximately the same as the phase velocity of the Langmuir wave under the modified condition. Therefore, it is concluded that $R_{2}$ and $E_{2}$ are excited due to the second $\mathrm{Lb}^{-}$ instability. The second acoustic decay instability is hardly recognized because of the strong Landau damping of the acoustic wave. The Landau damping effectively suppresses the acoustic decay instability since $v_{\mathrm{s}} \sim v_{\text {th }}$, whereas the $\mathrm{Lb}^{-}$ instability is less affected by the Landau damping because the phase velocity of the Langmuir wave is far from $v_{\text {th }}$.

\section{SUMMARY AND DISCUSSIONS}

We studied the linear dispersion relation of the parametric instabilities of the circularly polarized Alfvén wave in the relativistic and nonrelativistic electron-positron plasmas. The 
(a)

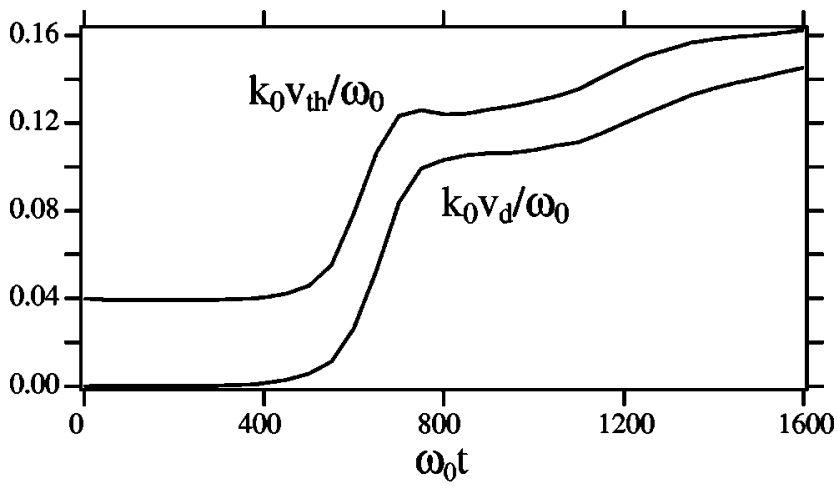

(b)

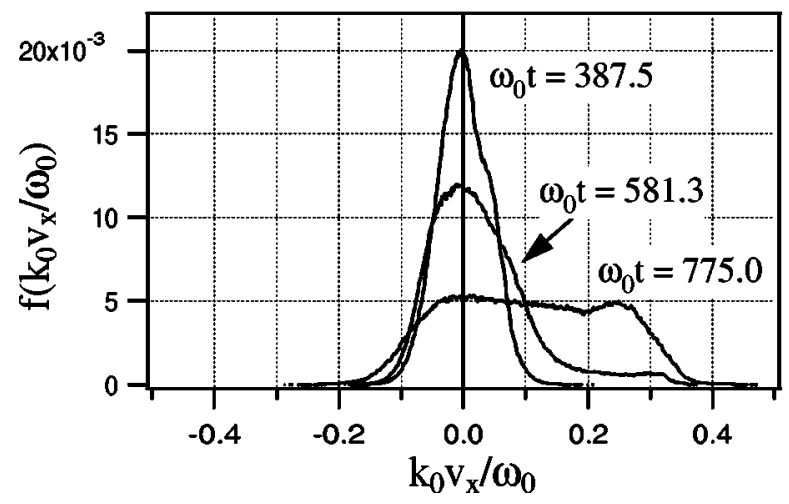

FIG. 12. (a) Time evolution of electron parallel drift and thermal velocities and (b) electron parallel distribution functions at $\omega_{0} t$ $=387.5,581.3$, and 775.0.

analytical expressions of the maximum growth rates of each instability are obtained in the nondispersive plasma, and the numerical solutions of the dispersion relations are shown for a variety of parameters both in the dispersive and nondispersive plasmas.

In the electron-positron plasma, the finite space charge effect is essential if $2 \omega_{\mathrm{p}}^{2}<1$. This condition can be realized in many astrophysical environments (in the electron-ion plasma, usually $\omega_{\mathrm{pe}}^{2} \gg \Omega_{\mathrm{i}}^{2}$, where $\omega_{\mathrm{pe}}, \Omega_{\mathrm{i}}$ are the the electron plasma and ion cyclotron frequencies). Moreover, the displacement current cannot be neglected in the high energy astrophysical plasma, since the Alfvén speed can often be close to the light speed. Table II summarizes the types of the instabilities we discussed in the nonrelativistic plasma.

In the no space charge limit $\left(\omega_{\mathrm{p}}^{2} \rightarrow \infty\right)$, the characteristics of the instability are basically the same as those in the electron-ion plasma, except that the modulational instability takes place only for $v_{\mathrm{s}}^{2}<1$ in the electron-positron plasma. On the other hand, the effect of the space charge cannot be ignored if $0<2 \omega_{\mathrm{p}}^{2}<1$. The $\mathrm{Lb}^{-}$and $\mathrm{Lf}^{-}$instabilities appear in this case. Although the $\mathrm{Lf}^{-}$instability is observed when $1<2 \omega_{\mathrm{p}}^{2}<\left(1+\Omega_{0}\right)^{2}$ and dispersive effect is neglected, it may be stable in reality. In fact, we could not find the instability by numerically solving Eq. (27), even for $\Omega_{0}^{2} \gg 1$, which includes the dispersive effect. Only the Alfvén beat instability is unstable regardless of the values of $\omega_{\mathrm{p}}^{2}$ when $v_{\mathrm{s}}^{2}>1$, although the maximum growth rate is small. At the same time, the acoustic decay instability is dominant for $v_{\mathrm{s}}^{2}$ $<1$ in a wide parameter regime. In addition, if $0<2 \omega_{\mathrm{p}}^{2}$ $<1$, the $\mathrm{Lb}^{-}$instability also has the maximum growth rate comparable with the acoustic decay instability.

In the linear analysis, we have not taken into account the kinetic effects of the plasma. They may be essential if $v_{\mathrm{s}}^{2}$ $>1$. We suspect that the growth rate of the Alfvén beat instability would be less due to the Landau damping should the formulation be made kinetically. (The kinetic effects on the acoustic decay instability in the electron-ion plasma are investigated in detail by Inhester [35].) Besides, in the high energy astrophysical plasma, $v_{\mathrm{s}}^{2}<c^{2} \approx 1$. This is why we have mainly discussed the case $v_{\mathrm{s}}^{2}<1$. However, as mentioned in the simulation section, the effect of the Landau damping for the acoustic decay instability may not be neglected even in this case, since $v_{\mathrm{s}} \sim v_{\text {th }}$ in the electronpositron plasma. In contrast, the phase velocity of the density perturbation of the $\mathrm{Lb}^{-}$instability is not close to $v_{\text {th }}$, so it may be possible that the parameter range where the $\mathrm{Lb}^{-}$ instability is dominant is wide when $0<2 \omega_{\mathrm{p}}^{2}<1$.

The weakly relativistic effect reduces the effective acoustic speed, the plasma frequency, and the effect of the parent amplitude. As a result, most of the instabilities, except for the acoustic decay instability for $v_{\mathrm{s}}>1 / 3$, are suppressed. In order to make more accurate analyses on the Alfvén beat instability, higher order correction terms representing the relativistic effect should be included. In addition, we have assumed that the plasma temperature is in a nonrelativistic regime. However, in astrophysical applications, the effects due to relativistically high plasma internal energy may be essentially important. Furthermore, as increasing the amplitude of the parent wave, in reality, the cyclotron frequency decreases as $\Omega_{0} \rightarrow \Omega_{0} / \gamma$. This effect should be included in dispersion analysis in the fully relativistic plasma in our future discussions.

TABLE II. Types of the instabilities in the nonrelativistic plasma.

\begin{tabular}{|c|c|c|c|c|c|c|}
\hline & \multicolumn{2}{|c|}{$0<2 \omega_{\mathrm{p}}^{2}<1$} & \multicolumn{2}{|c|}{$1<2 \omega_{\mathrm{p}}^{2}<\left(1+\Omega_{0}\right)^{2}$} & \multicolumn{2}{|c|}{$\left(1+\Omega_{0}\right)^{2}<2 \omega_{\mathrm{p}}^{2}\left(\omega_{\mathrm{p}}^{2} \rightarrow \infty\right)$} \\
\hline & $1 / \Omega_{0}^{2}=0$ & $1 / \Omega_{0}^{2} \neq 0$ & $1 / \Omega_{0}^{2}=0$ & $1 / \Omega_{0}^{2} \neq 0$ & $1 / \Omega_{0}^{2}=0$ & $1 / \Omega_{0}^{2} \neq 0$ \\
\hline$v_{\mathrm{s}}^{2}<1$ & $\begin{array}{c}\mathrm{AD}, \mathrm{Lb}^{-}, \\
\mathrm{Lf}^{-}\end{array}$ & $\begin{array}{c}\mathrm{AD}, \mathrm{Lb}^{-}, \\
\mathrm{AB}, \mathrm{Lf}^{-}, \\
M\end{array}$ & $\begin{array}{c}\mathrm{AD}, \mathrm{AB} \\
\left(\mathrm{Lf}^{-}, \text {see text }\right)\end{array}$ & $\begin{array}{c}\mathrm{AD}, \mathrm{AB}, \\
M\end{array}$ & $\mathrm{AD}$ & $\begin{array}{c}\mathrm{AD}, \mathrm{AB}, \\
\quad M\end{array}$ \\
\hline$v_{\mathrm{s}}^{2}>1$ & $\mathrm{AB}$ & $\mathrm{AB}$ & $\mathrm{AB}$ & $\mathrm{AB}$ & $\mathrm{AB}$ & $\mathrm{AB}$ \\
\hline
\end{tabular}


The nonlinear evolution of the system has been studied by using the one-dimensional full particle simulation code. In addition to the waves expected by the linear theory, various waves are excited by the beating of waves and by the successive decay via the $\mathrm{Lb}^{-}$instability. Decay instabilities both via the acoustic wave and via the Langmuir wave are observed. It should be emphasized here that the acoustic wave is easily Landau damped, while the Langmuir wave can survive. It is known, in general, that the daughter waves are unstable for further decay when they still have a large amplitude (see Terasawa et al. [13]). However, if the Landau damping is strong enough, the acoustic decay instability ceases even if the system still has the free energy (see Fig. 13). On the other hand, the $\mathrm{Lb}^{-}$instability can successively occur even if the plasma are heated, since the phase velocity of the Langmuir wave is far from $v_{\text {th }}$. Successive decay of Alfvén waves via the Langmuir wave channel provides a plausible candidate for an efficient generation of plasma turbulence.

We should note that our present simulation has not yet reached the final state, because of the slow evolution of the system due to insufficient energy of the parent wave. Hence, the simulation with higher energy should be performed for longer running time in the near future, using one- (or more) dimensional particle code. The particle acceleration is closely related with generation of the turbulence. In a high (a)

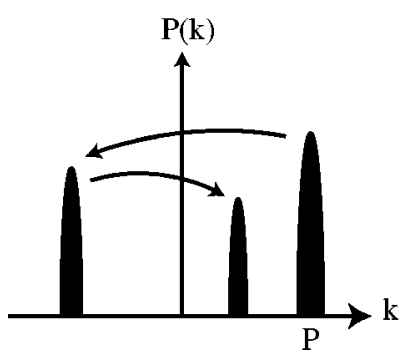

(b)

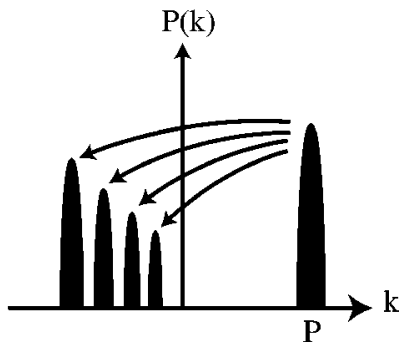

FIG. 13. Schematic picture of the successive decay processes via (a) the acoustic decay and (b) the $\mathrm{Lb}^{-}$instabilities. Although the successive acoustic decay is stopped due to the Landau damping of the acoustic waves, the Landau damping affects less the Langmuir waves.

energy system, in particular, the relativistic effects may boost production of energetic particles, since the low frequency waves excited due to the inverse cascading process preferentially interact with heavy (high energy) particles.

\section{ACKNOWLEDGMENTS}

We sincerely thank V. V. Krasnosel'skikh, B. Lembege, B. Lefebvre, and A. C.-L. Chian for valuable discussions. This paper has been financially supported by JSPS in Japan.

[1] S. Spangler, S. Fuselier, A. Fey and G. Anderson, J. Geophys. Res. [Space Phys.] 93, 845 (1988).

[2] S.R. Spangler, J.A. Leckband, and I.H. Cairns, Phys. Plasmas 4, 846 (1997).

[3] A.A. Galeev and V.N. Oraevskii, Dokl. Akad. Nauk (SSSR) 9 , 154 (1964) [Sov. Phys. Dokl. 7, 988 (1963)].

[4] R. Z. Sagdeev and A. A. Galeev, Nonlinear Plasma Theory (Benjamin, New York, 1969).

[5] M.L. Goldstein, Astrophys. J. 219, 700 (1978).

[6] N.F. Derby, Jr., Astrophys. J. 224, 1013 (1978).

[7] J. Mio, T. Ogino, K. Minami, and S. Takeda, J. Phys. Soc. Jpn. 41, 265 (1976).

[8] J. Mio, T. Ogino, K. Minami, and S. Takeda, J. Phys. Soc. Jpn. 41, 667 (1976).

[9] E. Mjolhus, J. Plasma Phys. 16, 321 (1976).

[10] J.I. Sakai and B.U.O. Sonnerup, J. Geophys. Res. [Space Phys.] 88, 9069 (1983).

[11] M. Longtin and B.U.O. Sonnerup, J. Geophys. Res. [Space Phys.] 91, 6816 (1986).

[12] H.K. Wong and M.L. Goldstein, J. Geophys. Res. [Space Phys.] 91, 5617 (1986).

[13] T. Terasawa, M. Hoshino, J.I. Sakai, and T. Hada, J. Geophys. Res. [Space Phys.] 91, 4171 (1986).

[14] V. Jayanti and J.V. Hollweg, J. Geophys. Res. [Space Phys.] 98, 19049 (1993).

[15] J.V. Hollweg, J. Geophys. Res. [Space Phys.] 99, 23431 (1994)

[16] A.F. Vinas and M.L. Goldstein, J. Plasma Phys. 46, 107 (1991).
[17] A.F. Vinas and M.L. Goldstein, J. Plasma Phys. 46, 129 (1991).

[18] S. Ghosh, A.F. Vinas, and M.L. Goldstein, J. Geophys. Res. [Space Phys.] 98, 15561 (1993).

[19] S. Ghosh, A.F. Vinas, and M.L. Goldstein, J. Geophys. Res. [Space Phys.] 99, 19289 (1994).

[20] S. Machida, S.R. Spangler, and C.K. Geortz, J. Geophys. Res. [Space Phys.] 92, 7413 (1987).

[21] S.R. Spangler and J.P. Sheerin, J. Plasma Phys. 27, 193 (1982).

[22] S.R. Spangler and J.P. Sheerin, Astrophys. J. 272, 273 (1983).

[23] S.R. Spangler, Phys. Fluids 29, 2535 (1986).

[24] S.R. Spangler, Phys. Fluids B 1, 1738 (1989).

[25] S.R. Spangler, Phys. Fluids B 2, 407 (1990).

[26] E. Mjolhus and J. Wyller, J. Plasma Phys. 40, 299 (1988).

[27] M. Hoshino and M.L. Goldstein, Phys. Fluids B 1, 1405 (1989).

[28] J. Arons and J.J. Barnard, Astrophys. J. 302, 120 (1986).

[29] D.R. Goncalves, V. Jatenco-Pereira, and R. Opher, Astron. Astrophys. 279, 351 (1993).

[30] G.S.S. Sweeney and P. Stewart, Astron. Astrophys. 66, 139 (1978).

[31] G.Z. Machabeli, S.V. Vladimirov, and D.B. Melrose, Phys. Rev. E 59, 4552 (1999).

[32] P.H. Yoon and L.F. Ziebell, Astrophys. J. 459, 529 (1996).

[33] V. Muñoz and L. Gomberoff, Phys. Rev. E 57, 994 (1998).

[34] T. Dudok de Wit and V.V. Krasnosel'skikh, Phys. Plasmas 2, 4307 (1995).

[35] B. Inhester, J. Geophys. Res. [Space Phys.] 95, 10525 (1990). 